\title{
Comment on: "Comparison of intracorporeal and extracorporeal anastomosis and resection in right colectomy: a systematic review and meta-analysis"
}

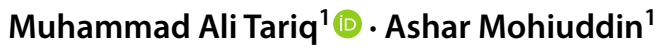

Received: 29 September 2021 / Accepted: 17 December 2021 / Published online: 29 January 2022

(c) The Author(s), under exclusive licence to Springer-Verlag GmbH Germany, part of Springer Nature 2021

\section{Dear Editor,}

We recently read the meta-analysis by Zheng et al. titled "Comparison of intracorporeal and extracorporeal anastomosis and resection in right colectomy: a systematic review and meta-analysis" published in the September issue of Langenbeck's Archives of Surgery [1]. The authors are commended for a well-written paper involving 30 studies with 4317 participants. However, we would like to communicate some of the methodological concerns regarding this paper.

Firstly, the authors utilized the Newcastle-Ottawa scale for quality assessment of all the included studies in this meta-analysis. The Newcastle-Ottawa scale was developed to appraise the quality of observational studies; therefore, it is inappropriate to use it for randomized control trials (RCTs) [2]. We suggest the authors to separately assess the quality of randomized control trials using the Cochrane Collaboration's Risk of Bias (ROB) Tool [3]. Secondly, as described in the Cochrane Handbook for Systematic Reviews, RCTs and observational studies cannot be combined unless the results are related to the harmful/adverse effects of the intervention [4]. Therefore, it is a methodological error to combine RCTs with observational studies in this meta-analysis. A better approach is to make a subgroup analysis of RCTs and observational studies for all the outcomes. Thirdly, high heterogeneity was observed in results of several study outcomes (surgical duration $I^{2}=83 \%$, intraoperative blood loss $I^{2}=81 \%$, number of harvested lymph nodes $I^{2}=71 \%$, incision length $I^{2}=95 \%$, hospitalization duration $I^{2}=87 \%$, time to first fluid $I^{2}=98 \%$, and time to first defecation $I^{2}=98 \%$ ), thus affecting the reliability of conclusions reached. Sadly, the authors did not discuss the

Muhammad Ali Tariq

m.alitariqpk@gmail.com

1 Department of Surgery, Dow University Hospital, Dow International Medical College, Suparco Road, Karachi, Pakistan implications of high heterogeneity on overall results of this meta-analysis. Possible sources of heterogeneity should be explored by sensitivity analyses and meta-regression.

Furthermore, the authors mention that they utilized the methodology suggested by Hozo et al. to estimate mean and standard deviation for studies where median with range was provided [5]. A major limitation of this method is that it assumes the outcome variable is normally distributed, which may be unlikely because otherwise the authors would have reported the data as mean with standard deviation and studies typically report the sample median and other sample quantiles when data are skewed. Therefore, we recommend the authors to use methods as proposed by McGrath et al. to estimate the sample mean and standard deviation for skewed data when the underlying distribution is unknown [6]. Lastly, recognition of publication bias is an important step in a meta-analysis. In this study, the authors concluded that there is absence of publication bias based on subjective visual inspection of the funnel plot. A better approach would be to use statistical test such as the Egger regression test to test for funnel plot asymmetry which would provide a better analysis for possible publication bias [7].

Once again, the authors are to be congratulated on their findings. We hope the authors will address the concerns raised, as this will only serve to strengthen the credibility of the conclusions reported in this meta-analysis.

\section{Declarations}

Ethics approval and consent to participate Not applicable.

Consent for publication Not applicable.

Competing interests The authors declare no competing interests. 


\section{References}

1. Zheng J-C, Zhao S, Chen W, Tang Y, Wang Y-Y, Wu J-X (2021) Comparison of intracorporeal and extracorporeal anastomosis and resection in right colectomy: a systematic review and metaanalysis. Langenbeck's Arch Surg 406(6):1789-1801

2. Farrah K, Young K, Tunis MC, Zhao L (2019) Risk of bias tools in systematic reviews of health interventions: an analysis of PROSPERO-registered protocols. Syst Rev 8(1):280. https://doi.org/10. 1186/s13643-019-1172-8

3. Sterne JAC, Savović J, Page MJ, Elbers RG, Blencowe NS, Boutron I et al (2019) RoB 2: a revised tool for assessing risk of bias in randomised trials. BMJ. 366:14898

4. Cumpston M, Li T, Page MJ, Chandler J, Welch VA, Higgins JP et al (2019) Updated guidance for trusted systematic reviews: a new edition of the Cochrane Handbook for Systematic Reviews of Interventions. Cochrane Database Syst Rev 10:ED000142
5. Hozo SP, Djulbegovic B, Hozo I (2005) Estimating the mean and variance from the median, range, and the size of a sample. BMC Med Res Methodol 5:13

6. McGrath S, Zhao X, Steele R, Thombs BD, Benedetti A (2020) Estimating the sample mean and standard deviation from commonly reported quantiles in meta-analysis. Stat Methods Med Res [Internet] 29(9):2520-2537

7. Egger M, Smith GD, Schneider M, Minder C (1997) Bias in meta-analysis detected by a simple, graphical test. BMJ 13 315(7109):629 LP - 634

Publisher's note Springer Nature remains neutral with regard to jurisdictional claims in published maps and institutional affiliations. 